\title{
Optimum Tuning of a Gyroscopic Vibration Absorber Using Coupled Gyroscopes for Vibration Control of a Vertical Cantilever Beam
}

\author{
F. Ünker ${ }^{1}$ and O. Çuvalcı ${ }^{2}$ \\ ${ }^{1}$ Department of Mechanical Engineering, Gümüşhane University, 29100 Gümüşhane, Turkey \\ ${ }^{2}$ Department of Mechanical Engineering, Karadeniz Technical University, 61100 Trabzon, Turkey \\ Correspondence should be addressed to F. Ünker; farukunker@gumushane.edu.tr
}

Received 28 September 2015; Accepted 20 December 2015

Academic Editor: Mickaël Lallart

Copyright (C) 2016 F. Ünker and O. Çuvalc1. This is an open access article distributed under the Creative Commons Attribution License, which permits unrestricted use, distribution, and reproduction in any medium, provided the original work is properly cited.

\begin{abstract}
This paper deals with the investigation of optimum values of the stiffness and damping which connect two gyroscopic systems formed by two rotors mounted in gimbal assuming negligible masses for the spring, damper, and gimbal support. These coupled gyroscopes use two gyroscopic flywheels, spinning in opposing directions to have reverse precessions to eliminate the forces due to the torque existing in the torsional spring and the damper between gyroscopes. The system is mounted on a vertical cantilever with the purpose of studying the horizontal and vertical vibrations. The equation of motion of the compound system (gyro-beam system) is introduced and solved to find the response measured on the primary system. This is fundamental to design, in some way, the dynamic absorber or neutralizer. On the other hand, the effect of the angular velocities of the gyroscopes are studied, and it is shown that the angular velocity (spin velocity) of a gyroscope has a significant effect on the behavior of the dynamic motion. Correctness of the analytical results is verified by numerical simulations. The comparison with the results from the derivation of the corresponding frequency equations shows that the optimized stiffness and damping values are very accurate.
\end{abstract}

\section{Introduction}

The attenuation of vibration caused by dynamic effects is desired in many engineering fields. To reduce or eliminate the undesirable motions, various types of structural control theories have been developed and evolved for different dynamic loads, and quite a few of them have been potentially practically useful. Structural control methods can be classified as passive, active, and semiactive control methods according to their energy consumption $[1,2]$. In the last decades, different types of structural control devices have been investigated for the possibility of using active and semiactive control methods to develop the control forces upon passive approaches for mitigating structural responses [2-7]. However, the passive control method is activated by the structural motion without requiring external force or energy to reduce structural responses and utilizes the motion of the structure at the location of the device [8]. Active control method requires considerable amount of external power to operate actuators that supply a control force to the structure. Due to requiring sensors and evaluator/controller equipment, active control devices are more complex, and, furthermore, they do not have reliability, low cost, and robustness compared to passive control techniques [6]. On the other hand, the active control is able to adapt to structural changes and to varying loading conditions.

The gyroscopic moment induced by a rotating object offers attractive means to protect structures against natural hazards in various ways. The engineering community have developed various stabilizer systems as a means for mitigating the effects of dynamic loading on structures. The use of gyrostabilizers has emerged, as they have an ability to control vibration at low frequency and represent a weight and volume saving, and, furthermore, the stored kinetic energy can provide emergency power [9-11]. For roll reduction, Dr. Schlick carried out such a device that has a high speed rotating 


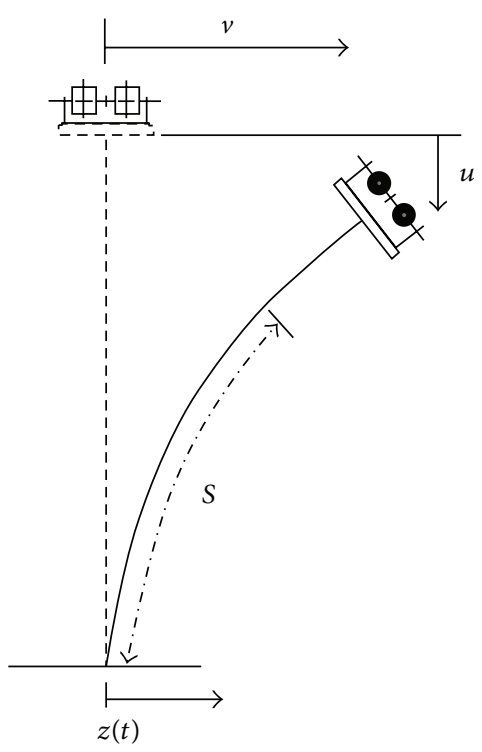

FIGURE 1: Cantilever model with end mass, coupled gyroscopes, and horizontal base excitation.

disk at a constant speed and yields producing a resistive gyroscopic torque as a source of angular momentum [12, 13]. The mechanism does not require any other external source of energy, in which the rotor speed is produced by an electric motor in a rotating gimbal. Therefore, this can be classified as a passive control device in a variation of passive vibration control systems. The gyrostabilizer is effective for bending moments rather than shear forces, because the gyrostabilizer utilizes the gyroscopic moment to reduce or eliminate the undesirable motions. Consequently, the purpose of this study is to evaluate a passive coupled-gyroscopic stabilizer as a modified form of Schlick's gyroscope. The problem of the Schlick gyroscope is solved by using an optimum torsional spring and damper to control the precession of the coupled gyroscopes that has also been developed for vibration mitigation of structures subjected to environmental and manmade loads. The coupled gyroscopes use two gyroscopic flywheels, spinning in opposing directions to have reverse precessions $\theta$ to eliminate the forces due to the torque existing in the torsional spring and the damper between gyroscopes. In this paper, the optimal values of the rotor speed, torsional spring, and damper are theoretically analyzed and derived for a cantilever beam. The rotor speed, the torsional spring, and the viscous damping for this gyrostabilizer are so tuned that this system is more adaptable and has smaller mass than other conventional control devices with the same ability to be employed for vibration control.

\section{Equations of Motion of the Gyros (Coupled)-Beam System for Small Vibration}

Here, the beam has mass density $\rho$, cross-sectional area $A$, equivalent Young's modulus $E$, moment of inertia of plane area $I$, and moment of inertia $I_{t}$ of a tip mass. Figure 1 shows a beam as a vertical cantilever with an end mass $M_{t}$ to which an additional gyro system (coupled gyroscopes with massless spring, damper, frame, and gimbal) is attached. The horizontal displacement of base subjected to a harmonic base excitation is $z$. The beam is assumed to be initially straight, of length $L$. The horizontal and vertical elastic displacements at the free end are $v$ and $u$, respectively. Due to elastic deformation of the beam, $s$ represents the distance along arclength of the beam.

The kinetic energy of the compound system (gyro-beam system) as shown in Figure 1 is

$$
\begin{aligned}
T= & \frac{1}{2} \rho A \int_{0}^{L}\left[(\dot{v}(s, t)+\dot{z}(t))^{2}+\dot{u}(s, t)^{2}\right] d s \\
& +\frac{1}{2} M_{t}\left[(\dot{v}(t)+\dot{z}(t))^{2}+\dot{u}(t)^{2}\right]+\frac{1}{2} I_{t} \dot{\varphi}^{2} \\
& +\frac{1}{2} m_{1}\left[(\dot{v}(t)+\dot{z}(t))^{2}+\dot{u}(t)^{2}\right] \\
& +\frac{1}{2} m_{2}\left[(\dot{v}(t)+\dot{z}(t))^{2}+\dot{u}(t)^{2}\right]+T_{g 1}+T_{g 2},
\end{aligned}
$$

where the motion of a gyro can be described by Euler's angles $\theta$ and $\varphi$. It is not difficult to show that the kinetic energy of gyroscopes can be expressed as

$$
\begin{aligned}
& T_{g 1}=\frac{1}{2} I_{o 1}\left[\dot{\theta}_{1}^{2}+\left(\dot{\varphi} \cos \theta_{1}\right)^{2}\right]+\frac{1}{2} I_{p 1}\left(\Omega_{1}+\dot{\varphi} \sin \theta_{1}\right)^{2}, \\
& T_{g 2}=\frac{1}{2} I_{o 2}\left[\dot{\theta}_{2}^{2}+\left(\dot{\varphi} \cos \theta_{2}\right)^{2}\right]+\frac{1}{2} I_{p 2}\left(\Omega_{2}+\dot{\varphi} \sin \theta_{2}\right)^{2} .
\end{aligned}
$$

The potential energy of the compound system can be written as

$$
\begin{aligned}
V= & \frac{1}{2} E I \int_{0}^{L} q(s, t)^{2} d s-\rho A g \int_{0}^{L} u(s, t) d s-M_{t} g u(t) \\
& +\frac{1}{2} k\left(\theta_{1}-\theta_{2}\right)^{2}-m_{1} g u(t)-m_{2} g u(t)
\end{aligned}
$$




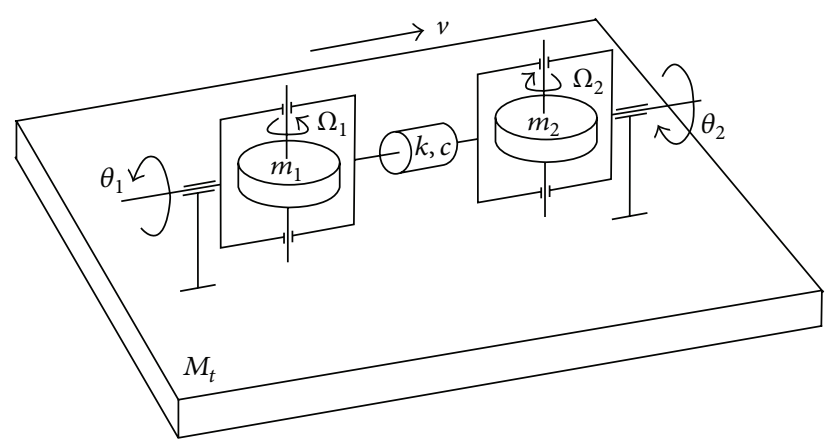

Figure 2: Coupled gyroscopes via a massless torsional spring with stiffness $k$ and a massless torsional damper with coefficient $c$.

The dissipation function $D$ takes the form

$$
D=\frac{1}{2} c\left(\dot{\theta}_{2}-\dot{\theta}_{1}\right)^{2}
$$

The gyro system consists of two disks, which can spin freely about their geometric axis via the massless gimbals mounted to the tip mass of the beam. The disk masses, $m$ of the gyros at free end, are assumed to have reverse angular speeds $\Omega$ and the precessions $\theta$ and also resisted by a torsional spring and damper torque defined by $k\left(\theta_{2}-\theta_{1}\right)$ and $c\left(\dot{\theta}_{2}-\dot{\theta}_{1}\right)$, respectively, as shown in Figure 2.

Equations of the beam and gyro have been expressed in equations (16), (17), and (18) in [14] as follows, respectively:

$$
\begin{aligned}
& \left\{\begin{array}{c}
\rho A G_{1}+m_{1}+m_{2}+M_{t}+I_{t} G_{5}^{2}+\left(\rho A G_{3}+\left(m_{1}+m_{2}+M_{t}\right) G_{4}^{2}+I_{t} G_{5}^{4}\right) v^{2}+\frac{1}{4} I_{t} G_{5}^{6} v^{4} \\
+\left(G_{5}+\frac{1}{2} v^{2} G_{5}^{3}\right)^{2}\left[I_{o 1}\left(\cos \theta_{1}\right)^{2}+I_{p 1}\left(\sin \theta_{1}\right)^{2}\right]+\left(G_{5}+\frac{1}{2} v^{2} G_{5}^{3}\right)^{2}\left[I_{o 2}\left(\cos \theta_{2}\right)^{2}+I_{p 2}\left(\sin \theta_{2}\right)^{2}\right]
\end{array}\right\} \ddot{v} \\
& +\left[\rho A G_{3}+\left(m_{1}+m_{2}+M_{t}\right) G_{4}^{2}+I_{t} G_{5}^{4}+\frac{1}{2} I_{t} G_{5}^{6} v^{2}\right] v \dot{v}^{2} \\
& +\left[E I G_{6}-\rho A g G_{9}-\left(m_{1}+m_{2}+M_{t}\right) g G_{4}+2 E I G_{7} v^{2}+\frac{3}{4} E I G_{8} v^{4}\right] v \\
& +\left(G_{5}+\frac{1}{2} v^{2} G_{5}^{3}\right)\left[\begin{array}{c}
\left(I_{p 1}-I_{o 1}\right)\left(\dot{v} G_{5}+\frac{1}{2} v^{2} \dot{v} G_{5}^{3}\right) \dot{\theta_{1}} \sin 2 \theta_{1} \\
+I_{o 1}\left(v \dot{v}^{2} G_{5}^{3}\right)\left(\cos \theta_{1}\right)^{2} \\
+I_{p 1}\left(v \dot{v}^{2} G_{5}^{3}\right)\left(\sin \theta_{1}\right)^{2} \\
+I_{p 1} \Omega_{1} \dot{\theta_{1}} \cos \theta_{1}
\end{array}\right] \\
& +\left(G_{5}+\frac{1}{2} v^{2} G_{5}^{3}\right)\left[\begin{array}{c}
\left(I_{p 2}-I_{o 2}\right)\left(\dot{v} G_{5}+\frac{1}{2} v^{2} \dot{v} G_{5}^{3}\right) \dot{\theta}_{2} \sin 2 \theta_{2} \\
+I_{o 2}\left(v \dot{v}^{2} G_{5}^{3}\right)\left(\cos \theta_{2}\right)^{2} \\
+I_{p 2}\left(v \dot{v}^{2} G_{5}^{3}\right)\left(\sin \theta_{2}\right)^{2} \\
+I_{p 2} \Omega_{2} \dot{\theta_{2}} \cos \theta_{2}
\end{array}\right]=-\left(\rho A G_{2}+m_{1}+m_{2}+M_{t}\right) \ddot{z} \\
& I_{o 1} \ddot{\theta}_{1}-\frac{1}{2}\left(I_{p 1}-I_{o 1}\right)\left(\dot{v} G_{5}+\frac{1}{2} v^{2} \dot{v} G_{5}^{3}\right)^{2} \sin 2 \theta_{1}-I_{p 1} \Omega_{1}\left(\dot{v} G_{5}+\frac{1}{2} v^{2} \dot{v} G_{5}^{3}\right) \cos \theta_{1}-c\left(\dot{\theta}_{2}-\dot{\theta}_{1}\right)-k\left(\theta_{2}-\theta_{1}\right)=0
\end{aligned}
$$




$$
I_{o 2} \ddot{\theta_{2}}-\frac{1}{2}\left(I_{p 2}-I_{o 2}\right)\left(\dot{v} G_{5}+\frac{1}{2} v^{2} \dot{v} G_{5}^{3}\right)^{2} \sin 2 \theta_{2}-I_{p 2} \Omega_{2}\left(\dot{v} G_{5}+\frac{1}{2} v^{2} \dot{v} G_{5}^{3}\right) \cos \theta_{2}+c\left(\dot{\theta}_{2}-\dot{\theta}_{1}\right)+k\left(\theta_{2}-\theta_{1}\right)=0
$$

where the constants from $G_{1}$ to $G_{9}$ are given in Table 2 .

After the rearrangement, (5) becomes

$$
\begin{aligned}
& \left\{\begin{array}{c}
\alpha_{1}+\alpha_{2} v^{2}+\alpha_{3} v^{4} \\
+\left(G_{5}+\frac{1}{2} v^{2} G_{5}^{3}\right)^{2}\left[\begin{array}{c}
I_{o 1}\left(\cos \theta_{1}\right)^{2}+I_{p 1}\left(\sin \theta_{1}\right)^{2} \\
+I_{o 2}\left(\cos \theta_{2}\right)^{2}+I_{p 2}\left(\sin \theta_{2}\right)^{2}
\end{array}\right]
\end{array}\right\} \ddot{v}+\left\{\alpha_{2}+2 \alpha_{3} v^{2}+G_{5}^{3}\left(G_{5}+\frac{1}{2} v^{2} G_{5}^{3}\right)\left[\begin{array}{c}
I_{o 1}\left(\cos \theta_{1}\right)^{2}+I_{p 1}\left(\sin \theta_{1}\right)^{2} \\
+I_{o 2}\left(\cos \theta_{2}\right)^{2}+I_{p 2}\left(\sin \theta_{2}\right)^{2}
\end{array}\right]\right\} v \dot{v}^{2} \\
& +\left[\alpha_{4}+\alpha_{5} v^{2}+\alpha_{6} v^{4}\right] v+\left(G_{5}+\frac{1}{2} v^{2} G_{5}^{3}\right)\left[\begin{array}{c}
\left(I_{p 1}-I_{o 1}\right) \dot{v}\left(G_{5}+\frac{1}{2} v^{2} G_{5}^{3}\right) \dot{\theta_{1}} \sin 2 \theta_{1} \\
+\left(I_{p 2}-I_{o 2}\right) \dot{v}\left(G_{5}+\frac{1}{2} v^{2} G_{5}^{3}\right) \dot{\theta_{2}} \sin 2 \theta_{2} \\
+I_{p 2} \Omega_{2} \dot{\theta}_{2} \cos \theta_{2} \\
+I_{p 1} \Omega_{1} \dot{\theta}_{1} \cos \theta_{1}
\end{array}\right]=-\gamma \ddot{z},
\end{aligned}
$$

in which

$$
\begin{aligned}
& \alpha_{1}=\rho A G_{1}+m_{1}+m_{2}+M_{t}+I_{t} G_{5}^{2}, \\
& \alpha_{2}=\rho A G_{3}+\left(m_{1}+m_{2}+M_{t}\right) G_{4}^{2}+I_{t} G_{5}^{4}, \\
& \alpha_{3}=\frac{1}{4} I_{t} G_{5}^{6}, \\
& \alpha_{4}=E I G_{6}-\rho A g G_{9}-\left(m_{1}+m_{2}+M_{t}\right) g G_{4}, \\
& \alpha_{5}=2 E I G_{7}, \\
& \alpha_{6}=\frac{3}{4} E I G_{8}, \\
& \gamma=\rho A G_{2}+m_{1}+m_{2}+M_{t} .
\end{aligned}
$$

Neglecting the terms of higher power for small vibrations $(v \approx$ $0),(6 a)$ and (6b) and (7) can be reduced to

$$
\begin{aligned}
& I_{o 1} \ddot{\theta}_{1}-\frac{1}{2}\left(I_{p 1}-I_{o 1}\right)\left(\dot{v} G_{5}\right)^{2} \sin 2 \theta_{1} \\
& -I_{p 1} \Omega_{1}\left(\dot{v} G_{5}\right) \cos \theta_{1}-c\left(\dot{\theta}_{2}-\dot{\theta}_{1}\right) \\
& \quad-k\left(\theta_{2}-\theta_{1}\right)=0, \\
& I_{o 2} \ddot{\theta}_{2}-\frac{1}{2}\left(I_{p 2}-I_{o 2}\right)\left(\dot{v} G_{5}\right)^{2} \sin 2 \theta_{2} \\
& \quad-I_{p 2} \Omega_{2}\left(\dot{v} G_{5}\right) \cos \theta_{2}+c\left(\dot{\theta}_{2}-\dot{\theta}_{1}\right) \\
& +k\left(\theta_{2}-\theta_{1}\right)=0,
\end{aligned}
$$

$$
\begin{aligned}
& \left\{+G_{5}^{2}\left[\begin{array}{c}
\alpha_{1}\left(\cos \theta_{1}\right)^{2}+I_{p 1}\left(\sin \theta_{1}\right)^{2} \\
+I_{o 2}\left(\cos \theta_{2}\right)^{2}+I_{p 2}\left(\sin \theta_{2}\right)^{2}
\end{array}\right]\right\} \ddot{v} \\
& +\left\{\alpha_{2}+G_{5}^{4}\left[\begin{array}{c}
I_{o 1}\left(\cos \theta_{1}\right)^{2}+I_{p 1}\left(\sin \theta_{1}\right)^{2} \\
+I_{o 2}\left(\cos \theta_{2}\right)^{2}+I_{p 2}\left(\sin \theta_{2}\right)^{2}
\end{array}\right]\right\} v \dot{v}^{2} \\
& +\alpha_{4} v+G_{5}\left[\begin{array}{c}
\left(I_{p 1}-I_{o 1}\right) \dot{v} G_{5} \dot{\theta_{1}} \sin 2 \theta_{1} \\
+\left(I_{p 2}-I_{o 2}\right) \dot{v} G_{5} \dot{\theta_{2}} \sin 2 \theta_{2} \\
+I_{p 2} \Omega_{2} \dot{\theta_{2}} \cos \theta_{2} \\
+I_{p 1} \Omega_{1} \dot{\theta_{1}} \cos \theta_{1}
\end{array}\right]=-\gamma \ddot{z} .
\end{aligned}
$$

\section{Optimal Tuning about the Equilibrium Position}

The easiest way to approach this problem is from the point of view of energy. We have chosen the kinetic to be zero when the tip mass is at the bottom of its swing. Setting $\dot{v} \approx 0$ and $\dot{\theta} \approx 0$ about the equilibrium position, (9a) and (9b) and (10) are reduced to

$$
\begin{aligned}
& I_{o 1} \ddot{\theta}_{1}-I_{p 1} \Omega_{1}\left(\dot{v} G_{5}\right) \cos \theta_{1}-c\left(\dot{\theta}_{2}-\dot{\theta}_{1}\right)-k\left(\theta_{2}\right. \\
& \left.\quad-\theta_{1}\right)=0, \\
& I_{o 2} \ddot{\theta}_{2}-I_{p 2} \Omega_{2}\left(\dot{v} G_{5}\right) \cos \theta_{2}+c\left(\dot{\theta}_{2}-\dot{\theta}_{1}\right)+k\left(\theta_{2}\right. \\
& \left.\quad-\theta_{1}\right)=0, \\
& \left\{\alpha_{1}+G_{5}^{2}\left[I_{o 1}\left(\cos \theta_{1}\right)^{2}+I_{p 1}\left(\sin \theta_{1}\right)^{2}+I_{o 2}\left(\cos \theta_{2}\right)^{2}\right.\right. \\
& \left.\left.\quad+I_{p 2}\left(\sin \theta_{2}\right)^{2}\right]\right\} \ddot{v}+\alpha_{4} v+G_{5}\left(I_{p 1} \Omega_{1} \dot{\theta_{1}} \cos \theta_{1}\right. \\
& \left.\quad+I_{p 2} \Omega_{2} \dot{\theta}_{2} \cos \theta_{2}\right)=-\gamma \ddot{z} .
\end{aligned}
$$


The natural frequencies of gyro and beam can be determined as follows, respectively:

$$
\begin{aligned}
& \omega_{g 1}^{2}=\frac{k}{I_{o 1}}, \\
& \omega_{g 2}^{2}=\frac{k}{I_{o 2}}, \\
& \omega_{n}^{2}=\frac{\alpha_{4}}{\left\{\alpha_{1}+G_{5}^{2}\left[I_{o 1}\left(\cos \theta_{1}\right)^{2}+I_{p 1}\left(\sin \theta_{1}\right)^{2}+I_{o 2}\left(\cos \theta_{2}\right)^{2}+I_{p 2}\left(\sin \theta_{2}\right)^{2}\right]\right\}} .
\end{aligned}
$$

Assume a harmonic disturbing base excitation $z=\bar{z} e^{j \omega t}$ and the responses may be written as angular frequency $\omega$ :

$$
\theta=\bar{\theta} e^{j \omega t}
$$

$$
v=\bar{v} e^{j \omega t}
$$

Therefore, (1la) and (11b) and (12) can be rewritten in the matrix form:

$$
\begin{aligned}
& \bar{\theta}_{1}\left[\begin{array}{c}
j \omega G_{5} I_{p 1} \Omega_{1} \cos \theta_{1} \\
-I_{o 1} \omega^{2}+j c \omega+k \\
-j c \omega-k
\end{array}\right]+\bar{\theta}_{2}\left[\begin{array}{c}
j \omega G_{5} I_{p 2} \Omega_{2} \cos \theta_{2} \\
-j c \omega-k \\
-I_{o 2} \omega^{2}+j c \omega+k
\end{array}\right]+\bar{v}\left[-\omega^{2}\left\{+G_{5}^{2}\left[\begin{array}{c}
\alpha_{o 1}\left(\cos \theta_{1}\right)^{2}+I_{p 1}\left(\sin \theta_{1}\right)^{2} \\
+I_{o 2}\left(\cos \theta_{2}\right)^{2}+I_{p 2}\left(\sin \theta_{2}\right)^{2}
\end{array}\right]\right\}+\alpha_{4}\right] \\
& =\left[\begin{array}{c}
\omega^{2} \gamma \bar{z} \\
0 \\
0
\end{array}\right] .
\end{aligned}
$$

The responses of gyro- 1 and gyro- 2 must become equal $\left(\left|\theta_{1}\right|=\right.$ $\left.\left|\theta_{2}\right|\right)$ when $I_{o 1}=I_{o 2}, I_{p 1}=I_{p 2}$, and $\Omega_{1}=-\Omega_{2}$. Hence, solving these equations yields

$$
\begin{aligned}
& \bar{v}=\frac{\omega^{2} \gamma \bar{z}\left[\left(-I_{o} \omega^{2}+j c \omega+k\right)^{2}-(-j c \omega-k)^{2}\right]}{x}, \\
& \bar{\theta}_{1} \\
& =\frac{\omega^{2} \gamma \bar{z}\left(-j \omega I_{p} \Omega G_{5} \cos \theta\right)\left[\left(-I_{o} \omega^{2}+j c \omega+k\right)-(-j c \omega-k)\right]}{x},
\end{aligned}
$$

where $x=\left\{\left(j \omega G_{5} I_{p} \Omega \cos \theta\right)^{2}\left[4(j c \omega+k)-2 I_{o} \omega^{2}\right]+\left[-\omega^{2}\left\{\alpha_{1}+\right.\right.\right.$ $\left.\left.2 G_{5}^{2}\left[I_{o}(\cos \theta)^{2}+I_{p}(\sin \theta)^{2}\right]\right\}+\alpha_{4}\right]\left[\left(-I_{o} \omega^{2}+j c \omega+k\right)^{2}-(-j c \omega-\right.$ $\left.\left.k)^{2}\right]\right\}$.

3.1. The Undamped Gyroscopic Vibration Absorber. By eliminating terms of damping, (17b) then yields

$$
\bar{\theta}_{2}=\frac{-\omega^{2} \gamma \bar{z}\left(-j \omega I_{p} \Omega G_{5} \cos \theta\right)\left(-I_{o} \omega^{2}+2 k\right)}{\left\{2\left(j \omega G_{5} I_{p} \Omega \cos \theta\right)^{2}\left(-I_{o} \omega^{2}+2 k\right)+\left[-\omega^{2}\left\{\alpha_{1}+2 G_{5}^{2}\left[I_{o}(\cos \theta)^{2}+I_{p}(\sin \theta)^{2}\right]\right\}+\alpha_{4}\right]\left(-I_{o} \omega^{2}\right)\left(-I_{o} \omega^{2}+2 k\right)\right\}} .
$$




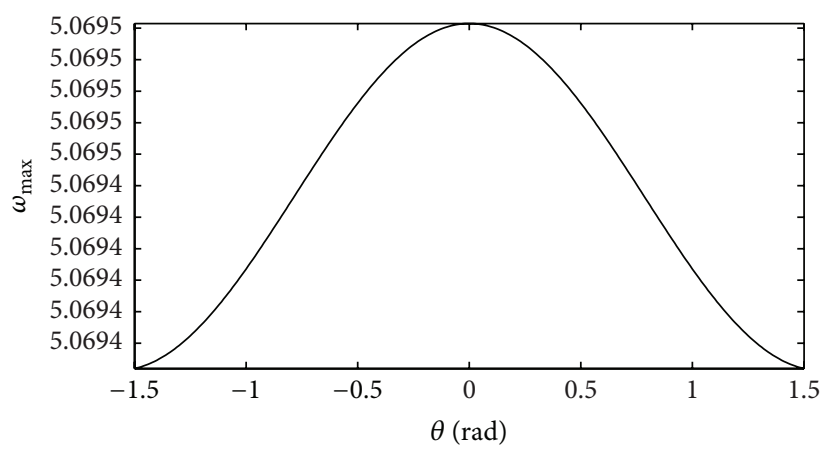

(a)

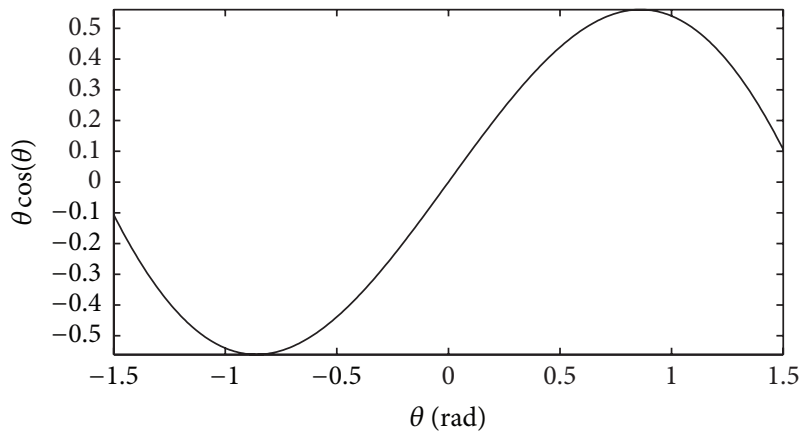

(b)

FIGURE 3: The theoretical limit curves: natural frequency rad/s (a); gyro response rad (b).

When $\omega^{2}=\omega_{n}^{2}$ and $\theta=\bar{\theta}_{2}=\bar{\theta}_{\text {max }}$ for equation of a stable motion at $\omega \leq \omega_{n}$ except for $\omega^{2}=k / I_{0}=\omega_{g}^{2}$, (18) corresponds to

$$
\left|\bar{\theta}_{\max }\right|=\frac{\gamma \bar{z} \omega_{n}}{2 G_{5} I_{p} \Omega \cos \bar{\theta}_{\max }} .
$$

Equation (19) can be modified into the following forms:

$$
\begin{aligned}
\bar{\theta}_{\max } \cos \bar{\theta}_{\max } & = \pm \frac{\omega_{n} \gamma \bar{z}}{2 I_{p} G_{5} \Omega}, \\
\omega_{\max } & = \pm \frac{2 I_{p} G_{5} \Omega \bar{\theta}_{\max } \cos \bar{\theta}_{\text {max }}}{\gamma \bar{z}} .
\end{aligned}
$$

The minimum disk speed $\Omega_{\min }$ should be chosen carefully to eliminate the instability at $\omega \leq \omega_{n}$. Suppose that a minimum disk speed $\Omega_{\min }$ is required for a stable motion. Then, this transforms to a minimum disk speed equation:

$$
\Omega_{\min }= \pm \frac{\omega_{n} \gamma \bar{z}}{2 I_{p} G_{5} \bar{\theta}_{\max } \cos \bar{\theta}_{\max }} .
$$

Figure 3 shows that the theoretical limit of the amplitude at resonance is one when absolute value of the precession $\bar{\theta}_{\max }$ approaches $0.86 \mathrm{rad}$. Suppose that $\left|\bar{\theta}_{\max } \cos \bar{\theta}_{\max }\right|=0.5611$ and $\left|\bar{\theta}_{\max }\right|=0.86 \mathrm{rad}$ for $-\pi / 2 \leq \bar{\theta} \leq \pi / 2$. Equation (21) may be rewritten as

$$
\Omega_{\min }=0.8911 \frac{\omega_{n} \gamma \bar{z}}{I_{p} G_{5}}
$$

where (13b) is then

$$
\omega_{n}=\sqrt{\frac{\alpha_{4}}{\alpha_{1}+2 G_{5}^{2}\left[0.426 I_{o}+0.574 I_{p}\right]}} .
$$

For the minimum tip mass displacement $|\bar{v}|=0$, the excitation frequency should be $\omega^{2}=2 k / I_{o}$ obtained from (16).

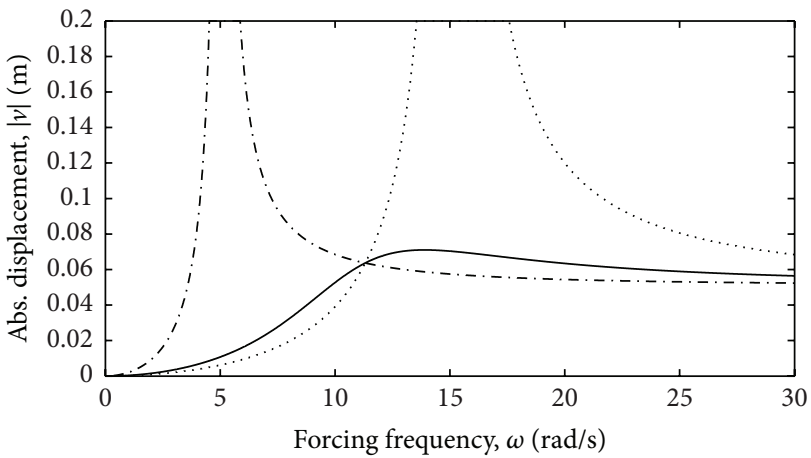

FIGURE 4: The theoretical frequency response curves of tip of beam with $\Omega_{\min }=15850 \mathrm{rpm}$ and $\bar{z}=0.05 \mathrm{~m}$; for (dotted) $k=0$ and $c=0$, for (dash-dot) $k=I_{o} \omega^{2} / 2$ and $c=0$, and for (solid) $k=I_{o} \omega^{2} / 2$ and $c=I_{o} \omega^{2} / 4 \omega_{g}$.

3.2. The Damped Gyroscopic Vibration Absorber. With the help of (16), in order to reach the minimum tip mass displacement $|\bar{v}|=0$, it can be rewritten as

$$
\omega^{2} \gamma \bar{z}\left[\left(-I_{o} \omega^{2}+j c \omega+k\right)^{2}-(-j c \omega-k)^{2}\right]=0 .
$$

Then, optimal damping can be obtained:

$$
c= \pm \frac{I_{o} \omega^{2}-2 k}{2 \omega} .
$$

Set $k=I_{o} \omega_{n}^{2} / 2$ and $\omega=\omega_{g}=\sqrt{k / I_{o}}$ to eliminate the instability due to the natural frequency of the added gyrospring system. Equation (25) is then

$$
c_{\mathrm{opt}}=\frac{I_{o} \omega_{n}^{2}}{4 \omega_{g}} .
$$

Figure 4 shows the possible maxima and minima of the theoretical frequency response at the tip of beam.

\section{Numerical Simulations}

In the following calculations, a rectangle-cross beam is considered with thickness $h=100 \mathrm{~mm}$, width $b=150 \mathrm{~mm}$, 
TABLE 1: Physical properties of the system.

\begin{tabular}{lcc}
\hline Symbol & Numerical values & Description \\
\hline$E$ & $210 \times 10^{9} \mathrm{~N} / \mathrm{m}^{2}$ & Young's modulus \\
$h$ & $100 \mathrm{~mm}$ & Beam thickness \\
$b$ & $150 \mathrm{~mm}$ & Beam width \\
$L$ & $5 \mathrm{~m}$ & Length of the beam \\
$\rho$ & $7850 \mathrm{~kg} / \mathrm{m}^{3}$ & Density of the beam \\
$M_{t}$ & $2000 \mathrm{~kg}$ & Tip mass \\
$I_{t} / M_{t}$ & $0.2 \mathrm{~m}{ }^{2}$ & Ratio of tip mass moment of inertia \\
$I_{t}$ & $400 \mathrm{~kg} \cdot \mathrm{m}^{2}$ & Tip mass moment of inertia \\
$I$ & $1.25 \times 10^{-5} \mathrm{~m}^{4}$ & Geometrical moment of inertia of beam \\
$g$ & $9.81 \mathrm{~m} / \mathrm{s}^{2}$ & Gravitational acceleration \\
$A=b \cdot h$ & $0.015 \mathrm{~m}$ & Area of cross section of beam \\
$c$ & $I_{o} \omega_{n}^{2} / 4 \omega_{g} \mathrm{~N} \cdot \mathrm{m}^{2} / \mathrm{rad}$ & Damping coefficient \\
$k$ & $I_{0} \omega_{n}^{2} / 2 \mathrm{~N} \cdot \mathrm{m} / \mathrm{rad}$ & Stiffness of torsion spring \\
$m=m_{1}=m_{2}$ & $50 \mathrm{~kg}$ & Disk mass of gyroscope \\
$r=r_{1}=r_{2}$ & $0.2 \mathrm{~m}$ & Radius of disk \\
$I_{p}=I_{p 1}=I_{p 2}=m \cdot r^{2} / 2$ & $1 \mathrm{~kg} \cdot \mathrm{m}^{2}$ & Rotary inertia of disk \\
$I_{o}=I_{o 1}=I_{o 2}=I_{p} / 2$ & $0.5 \mathrm{~kg} \cdot \mathrm{m}^{2}$ & Mass moment of inertia of disk \\
$\Omega=\Omega_{1}=-\Omega_{2}$ & $0-30000 \mathrm{rpm}$ & Rotating speed of disk
\end{tabular}

TABLE 2: Constants of the equation of motion of the gyro-beam system in SI units.

\begin{tabular}{ccccccccc}
\hline$G_{1}$ & $G_{2}$ & $G_{3}$ & $G_{4}$ & $G_{5}$ & $G_{6}$ & $G_{7}$ & $G_{8}$ & $G_{9}$ \\
\hline 1.1338 & 1.8169 & 0.0552 & 0.2467 & 0.3142 & 0.0244 & $6.0087 e-4$ & $2.9652 e-5$ & 0.3669 \\
\hline
\end{tabular}

length $L=5 \mathrm{~m}$, density $\rho=7850 \mathrm{~kg} / \mathrm{m}^{3}$, and Young's modulus along the axial direction $E=210 \times 10^{9} \mathrm{~N} / \mathrm{m}^{2}$. Equations may be solved by using a Matlab software tool that involves the fourth-order Runge-Kutta method. The parameters of the numerical example are given in Table 1. In order to identify the dynamical behavior, frequency response is simulated, with the time step size of $0.1 \mathrm{~s}$ and zero initial conditions.

4.1. The Undamped Gyroscopic Vibration Absorber. In the case of the base excitation with $z=0.05 \cos \left(\omega_{n} t\right)$, Figure 5 shows the rotational speed curve for case $k=I_{o} \omega_{n}^{2} / 2$. It can be seen that tip response of beam is effectively reduced over minimum rotational speed $\left(\Omega_{\min }=15850 \mathrm{rpm}\right)$.

Figure 6 represents the frequency response curve of the beam and gyro for cases $\Omega_{\min }=15850 \mathrm{rpm}$ and $k=I_{o} \omega_{n}^{2} / 2$. It presented that the displacement response is effectively reduced before the case $\omega=\omega_{n}$. But resonance occurs when $\omega=\omega_{n}$. However, over the resonance frequency, reduction of the vibration continues to reach the optimum scale at minimum rotational speed.

4.2. The Damped Gyroscopic Vibration Absorber. In the case of the base excitation with $z=0.05 \cos \left(\omega_{n} t\right)$, Figure 7 shows the rotational speed curve for cases $k=I_{o} \omega_{n}^{2} / 2$ and $c_{\text {opt }}=$ $I_{o} \omega_{n}^{2} / 4 \omega_{g}$. The response of the beam is effectively reduced and effect reaches low rotational speeds compared to undamped gyros. When damping coefficient is zero, the response of gyro can jump into unstable zone at resonance as seen in Figure 5.

It can be seen that the case of $c_{\text {opt }}=I_{o} \omega_{n}^{2} / 4 \omega_{g} \mathrm{~N} \cdot \mathrm{m} \cdot \mathrm{s} / \mathrm{rad}$ is an optimum damping coefficient, which effectively reduced the response of the beam at resonance shown in Figure 8. When optimum damping coefficient is chosen, the frequency response of the beam can improve compared to Figures 5 and 6; therefore, cases $k=I_{o} \omega_{n}^{2} / 2$ and $c_{\text {opt }}=I_{o} \omega_{n}^{2} / 4 \omega_{g}$ are chosen to reach the optimum scale for minimum rotational speed. As a result, it is shown that optimum values of the stiffness and damping which connect two gyroscopic systems have a significant effect on the the performance of the gyroscopic vibration absorber. On the other hand, the performance of the system is improved as we increase the rotor speed as shown in Figure 9.

\section{Conclusions}

The optimal parameters of the rotor speed, the spring, and damper for the minimum responses of the gyroscopes and beam are studied. This paper provides some analytical equations to investigate these optimal parameters of a gyrobeam system. The effectiveness is presented by computer simulations and the numerical results agree with the analytical solutions. From the consistency of the present study, the following conclusions are found:

(1) Up to a certain rotor speed there is no mitigation in the vibration of beam at free end. But beyond this, 


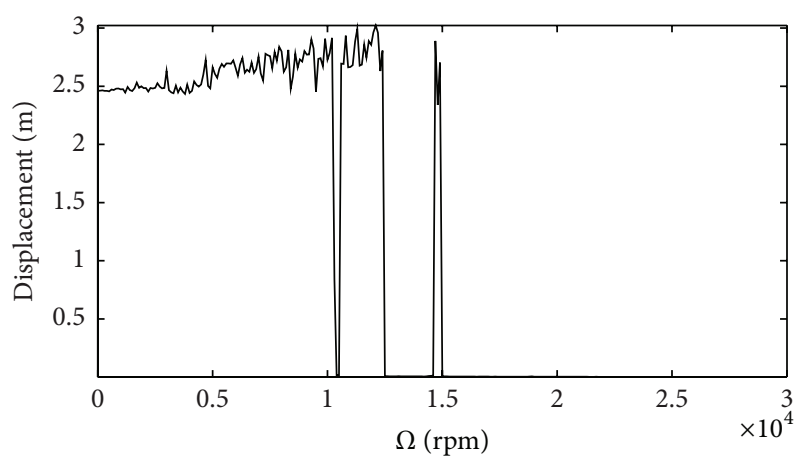

(a)

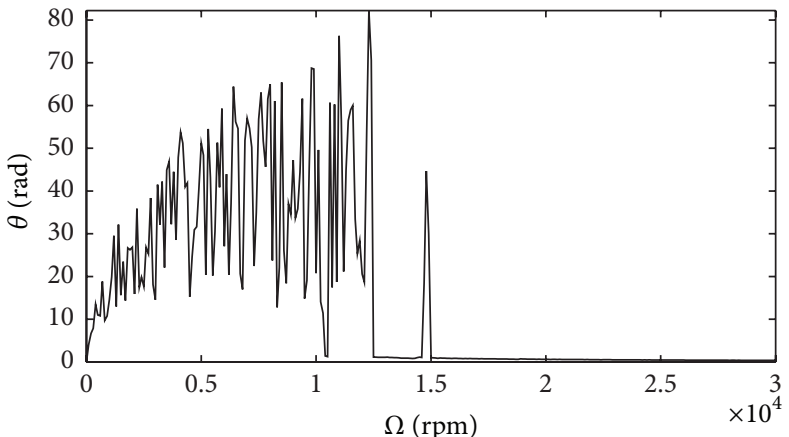

(b)

FIGURE 5: Rotational speed response curves: tip response of beam (a); gyro response (b) for $k=I_{o} \omega_{n}^{2} / 2=6.4 \mathrm{Nm} / \mathrm{rad}$ with the base excitation of $z=0.05 \cos \left(\omega_{n} t\right)$.

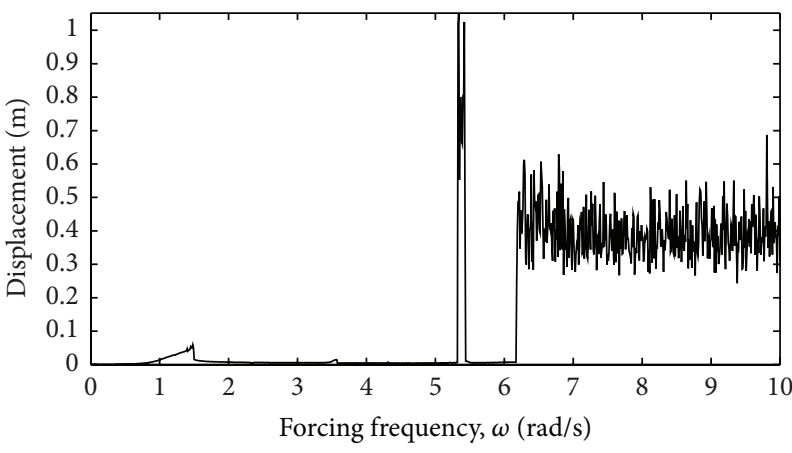

(a)

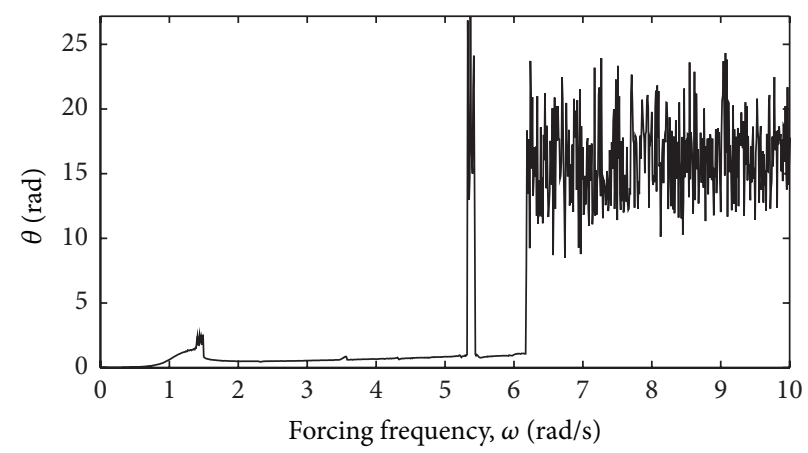

(b)

FIGURE 6: Frequency response curves for $\Omega_{\min }=15850 \mathrm{rpm}$ : tip response of beam (a); gyro response (b) for $k=I_{o} \omega_{n}^{2} / 2=6.4 \mathrm{Nm} / \mathrm{rad}$ with the base excitation of $z=0.05 \cos (\omega t)$.

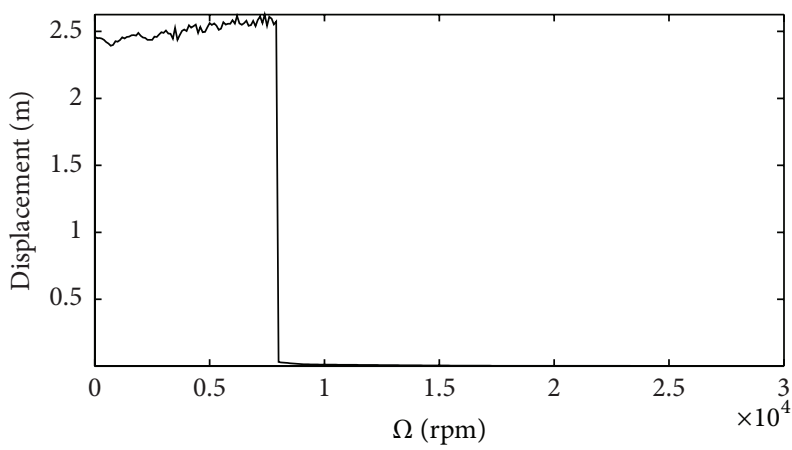

(a)

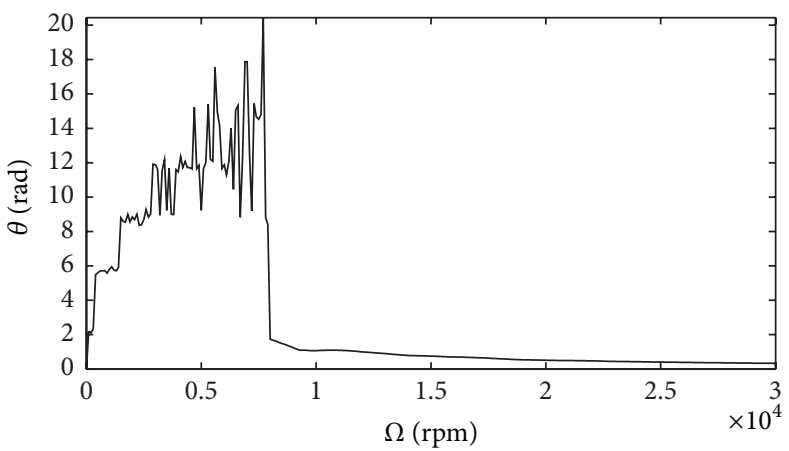

(b)

FIGURE 7: Rotational speed response curves: tip response of beam (a); gyro response (b) for $k=I_{o} \omega_{n}^{2} / 2=6.4 \mathrm{Nm} / \mathrm{rad}$ and $c_{\mathrm{opt}}=I_{o} \omega_{n}^{2} / 4 \omega_{g}=$ $0.9 \mathrm{Nms} / \mathrm{rad}$ with the base excitation of $z=0.05 \cos \left(\omega_{n} t\right)$.

as we increase the rotor speed, the gyro system has significantly reduced the vibration of beam. Hence this value is selected as the minimum rotor speed $\left(\Omega_{\min }=0.8911\left(\omega_{n} \gamma \bar{z} / I_{p} G_{5}\right)\right)$. The rotary inertia of the rotor can be reduced by increasing rotor speed, which is proportional to the mass of disk. Therefore, the weight and volume of the system can be reduced.
(2) There exist optimum parameters for the spring and damper for minimum response of the beam. The stiffness of the spring and damper affect the performance of gyroscopes. The gyroscopes can jump into unstable zone when the stiffness is not taken properly. Optimum spring and damper parameters for minimum response are obtained from frequency 


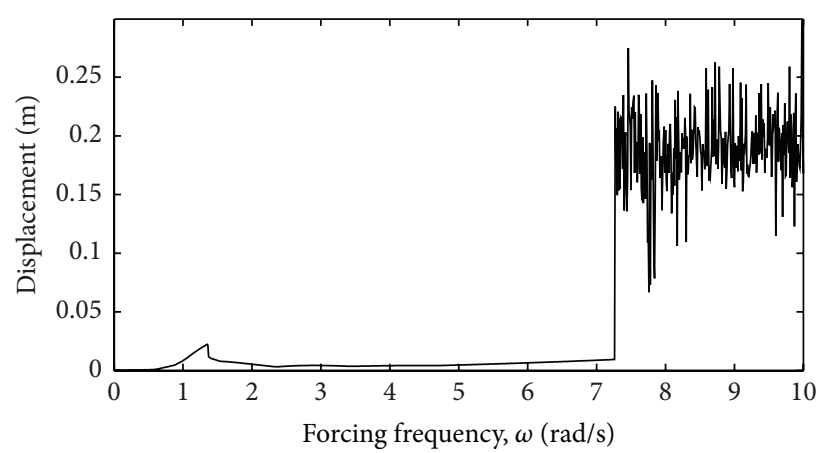

(a)

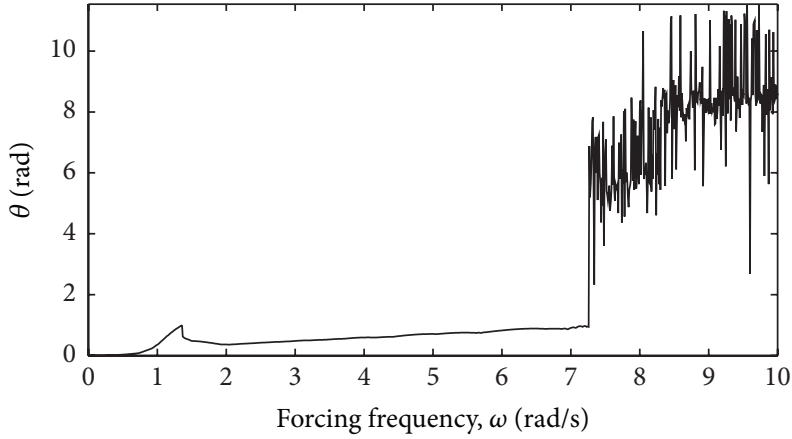

(b)

FIGURE 8: Frequency response curves for $\Omega_{\min }=15850 \mathrm{rpm}$ : tip response of beam (a); gyro response (b) for $k=I_{o} \omega_{n}^{2} / 2=6.4 \mathrm{Nm} / \mathrm{rad}$ and $c_{\mathrm{opt}}=I_{o} \omega_{n}^{2} / 4 \omega_{g}=0.9 \mathrm{Nms} / \mathrm{rad}$ with the base excitation of $z=0.05 \cos (\omega t)$.

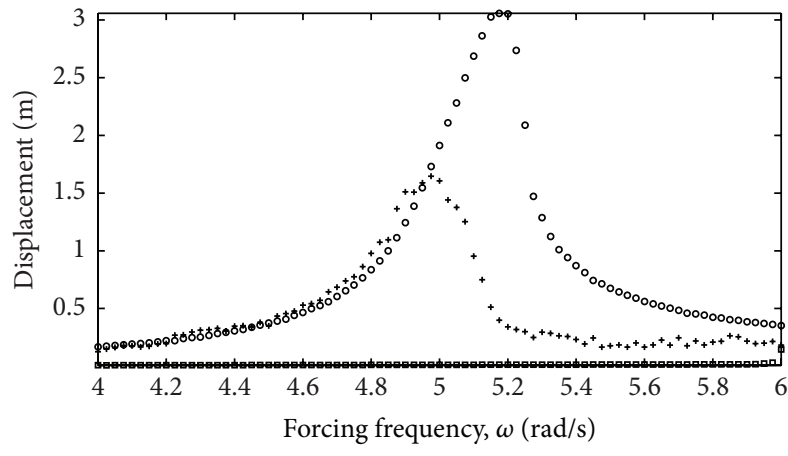

(a)

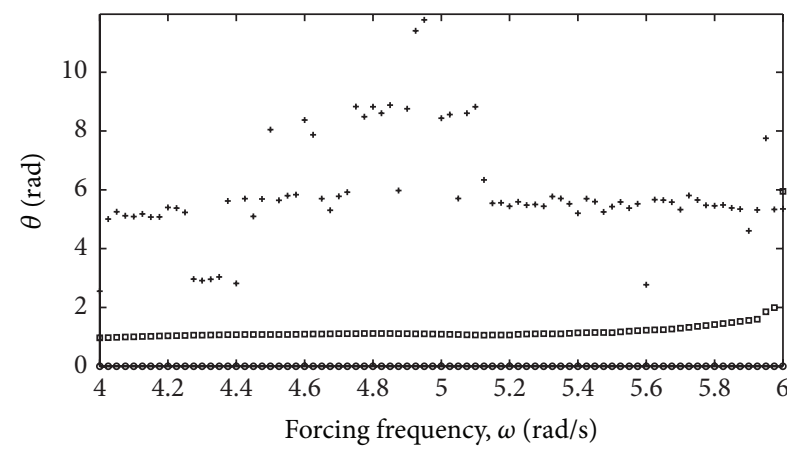

(b)

FiguRE 9: Frequency response curves for $k=I_{o} \omega_{n}^{2} / 2=6.4 \mathrm{Nm} / \mathrm{rad}$ and $c_{\mathrm{opt}}=I_{o} \omega_{n}^{2} / 4 \omega_{g}=0.9 \mathrm{Nms} / \mathrm{rad}$ : tip response of beam (a); gyro response (b) for (o) $\Omega=0 \mathrm{rpm},(+) \Omega=7500 \mathrm{rpm}$, and () $\Omega=10000 \mathrm{rpm}$ with the base excitation of $z=0.05 \cos (\omega t)$.

equation of beam. The minimum displacement having optimum spring coefficient should be chosen for optimal damper at the resonance frequency. Then, setting $\omega=\omega_{g}$ to eliminate the instability due to the natural frequency of the added gyro-spring system results in optimal damping. Coupling the gyroscopes with the optimal spring $\left(k=I_{o} \omega_{n}^{2} / 2\right)$ and damper $\left(c=I_{o} \omega_{n}^{2} / 4 \omega_{g}\right)$ is a practical and effective approach to mitigate the undesired motions of beam and gyroscopes.

\section{Conflict of Interests}

The authors declare that there is no conflict of interests regarding the publication of this paper.

\section{Acknowledgment}

This study was mainly supported by the Scientific and Technological Research Council of Turkey (TUBITAK) under Grant no. 114M760.

\section{References}

[1] T. K. Datta, "A state-of the-art review on active control of structures," ISET Journal of Earthquake Technology, Paper No. 430 , vol. 40 , no. 1 , pp. 1-17, 2003.

[2] G. W. Housner, L. A. Bergman, T. K. Caughey et al., "Structural control: past, present and future," Journal of Engineering Mechanics, vol. 123, no. 9, pp. 897-971, 1997.

[3] T. T. Soong, Active Structural Control: Theory and Practice, Longman Scientific and Technical, New York, NY, USA, 1990.

[4] B. F. Spencer Jr. and M. K. Sain, "Controlling buildings: a new frontier in feedback," IEEE Control Systems Magazine, vol. 17, no. 6, pp. 19-35, 1997.

[5] T. Kobori, "Past, present and future in seismic response control in civil engineering structures," in Proceedings of the 3rd World Conference on Structural Control, pp. 9-14, Wiley, New York, NY, USA, 2003.

[6] T. T. Soong and B. F. Spencer Jr., "Supplemental energy dissipation: state-of-the-art and state-of-the-practice," Engineering Structures, vol. 24, no. 3, pp. 243-259, 2002.

[7] B. F. Spencer Jr., "Civil engineering applications of smart damping technology," in Proceedings of the 5th International 
Conference on Vibration Engineering, pp. 771-782, Nanjing, China, 2002.

[8] T. T. Soong and G. F. Dargush, Passive Energy Dissipation System in Structural Engineering, Wiley, New York, NY, USA, 1997.

[9] N. C. Townsend, A. J. Murphy, and R. A. Shenoi, "A new active gyrostabiliser system for ride control of marine vehicles," Ocean Engineering, vol. 34, no. 11-12, pp. 1607-1617, 2007.

[10] B. Thornton, T. Ura, Y. Nose, and S. Turnock, "Internal actuation of underwater robots using control moment gyros," in Proceedings of the IEEE OCEANS Conference, vol. 1, pp. 591-598, Brest, France, June 2005.

[11] T. Perez and P. Steinmann, "Analysis of ship roll gyrostabiliser control," in Proceedings of the 8th IFAC Conference on Manoeuvring and Control of Marine Craft, pp. 310-315, Guarujá, Brazil, September 2009.

[12] E. O. Schlick, "Device for minimising the oscillatory movements of ships," Patent US769, 493, 1904.

[13] E. O. Schlick, "The gyroscopic effect of flywheels on board ship," Transactions of the Institute of Naval Architects, vol. 23, pp. 117134, 1904.

[14] F. Ünker and O. Çuvalcı, "Nonlinear motion control of a column using a coupled gyroscope," Procedia-Social and Behavioral Sciences, vol. 195, pp. 2242-2252, 2015. 


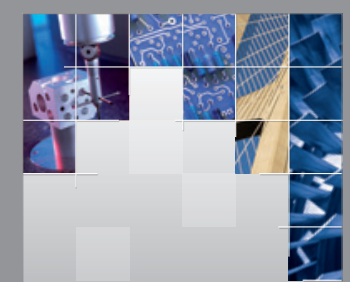

\section{Enfincering}
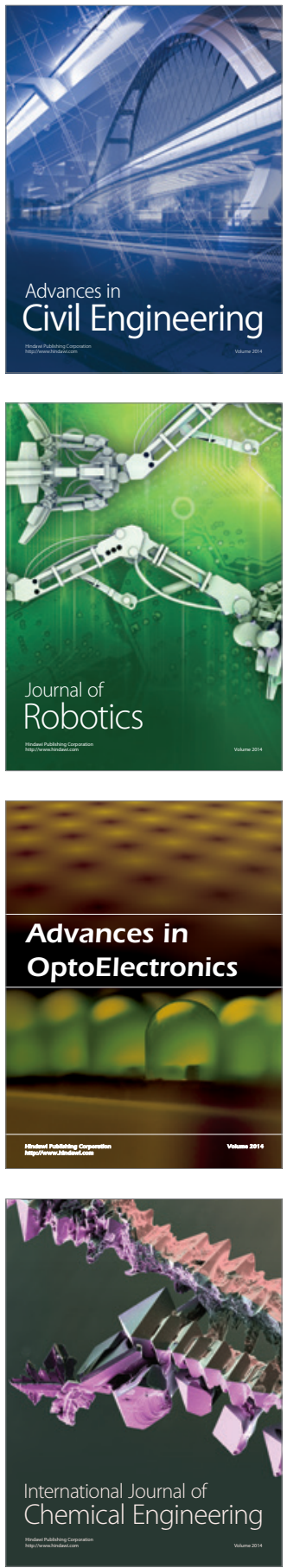

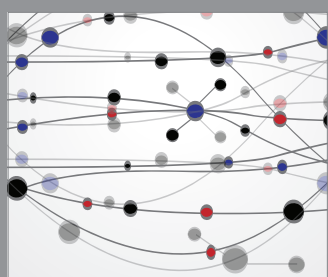

The Scientific World Journal

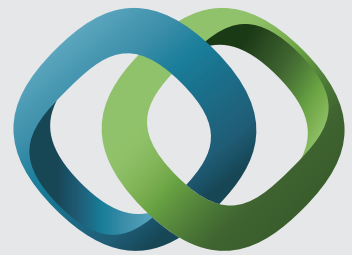

\section{Hindawi}

Submit your manuscripts at

http://www.hindawi.com
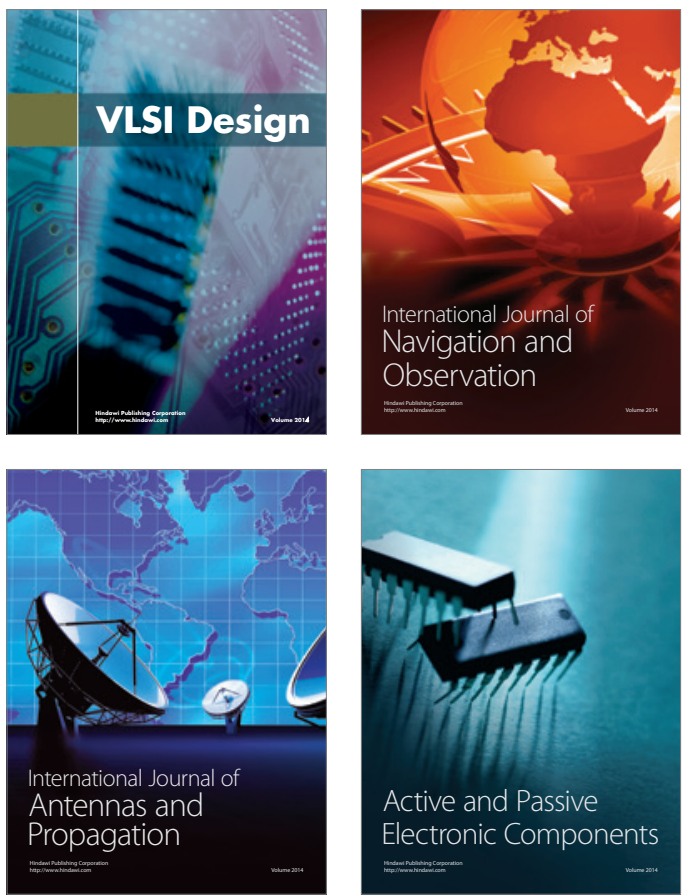
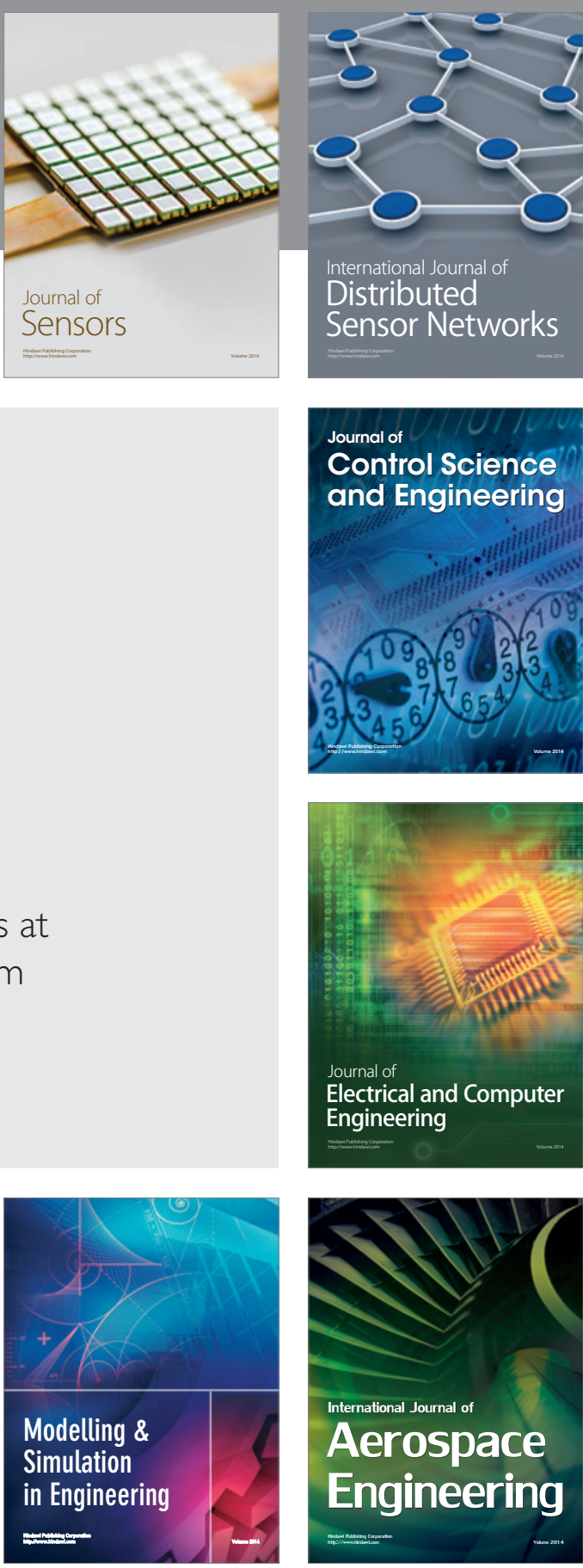

International Journal of

Distributed

Sensor Networks

Journal of

Control Science

and Engineering
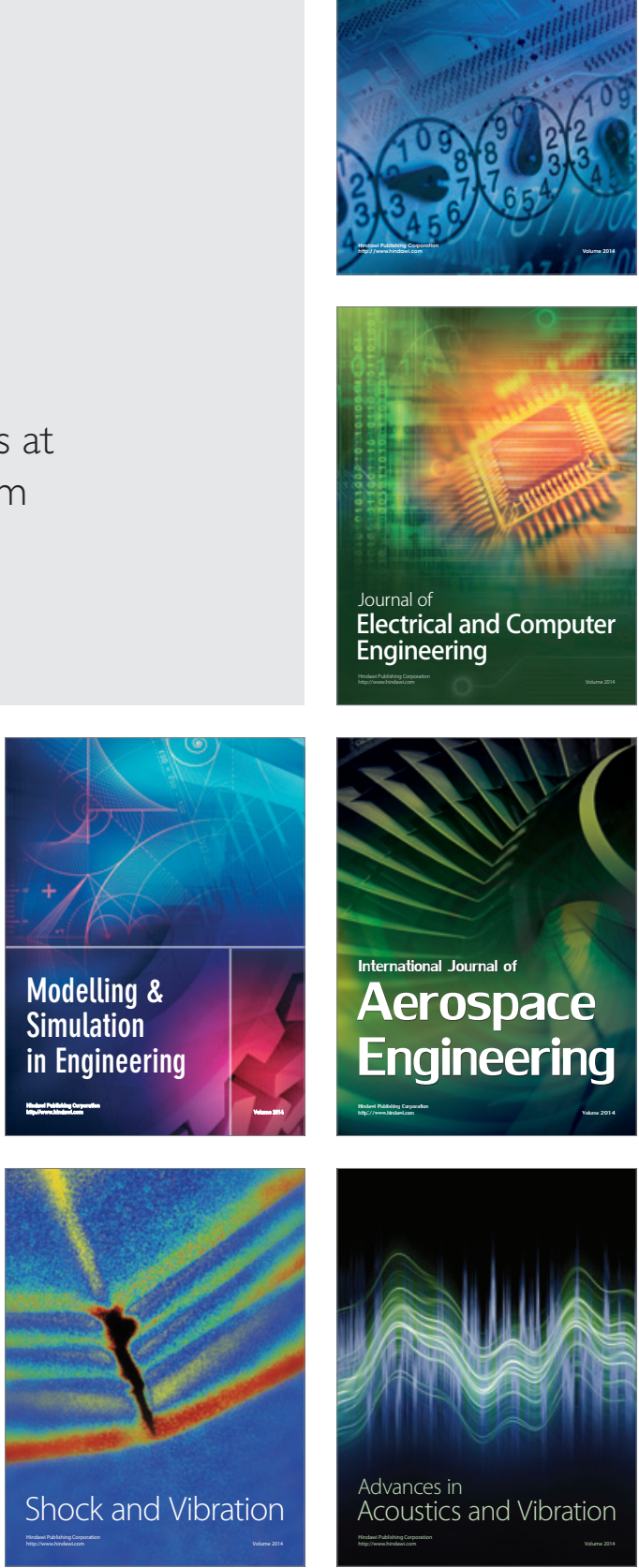